\title{
Correlation Between Tooth form, Face Form and Arch Form Using Computer Program
}

\author{
Dr R S Gugwad ${ }^{1}$, Dr. Savita Akki ${ }^{2}$, Dr Sharanbasappa C Nagaral ${ }^{3}$ \\ ${ }^{I}$ Professor And HOD, Dept Of Dentistry Bidar Institute Of Medical Sciences. Bidar- Karnataka State \\ ${ }^{2}$ Associate Professor, Department Of Dentistry, Bidar Institute Of Medical Sciences. \\ Bidar- Karnataka State, India. \\ ${ }^{3}$ Professor Dept Of Prosthodontics Al Badar Dental College, Gulbarga, Karnataka.
}

\begin{abstract}
Number of methods have been studied and suggested for selection of artificial tooth for determining the artificial tooth form to find out reliable esthetic factor. In this article a method is adopted to find out the esthetic factor by superimposition of the outline form of the tooth form(central incisor), arch fom and face outline. 50 male dental students were selected and impression of the maxillary arch is made using irreversible hydrocolloid material and cast formed. A standardized photographic procedure is used to get the face photo of each student. And then Photoshop computer program is used to get the outline form of the incisor tooth, face, and the upper arch form. and then super imposition of the all these outline is made and correlate these factor to find out esthetic factor. Result in this study shows there is insignificant correlation between face, tooth, and the arch form. And using this as esthetic guide is not advisable and impractical for common application.
\end{abstract}

Keywords: Artificial tooth, esthetic factor, tooth form, arch form, face form, superimposition, esthetic guide.

\section{Introduction}

The loss of natural teeth especially anterior natural teeth can be devasting way for some persons and their replacement with artificial substitute such as fixed partial denture ,Denture (Remorale) and implant may be necessary for restoration of esthetics and function. The choice of artificial teeth can be more difficult if the patients request is for reproduction of features which are prevent in natural dentition like tooth irregularities ,wear, staining .

Literature shows that several factors have been suggested as and for artificial tooth selection ${ }^{[1-4]}$ and numerous methods have been devised for the evaluation and reliable esthetic factors in determining artificial tooth form ${ }^{[5]}$ Suggestions have been made for alternative expedient guide to anterior tooth selection, such as facial contours in different plans and anatomic landmarks ${ }^{[7]}$ It was observed in previous studies the outline of the face, when inverted may correspond with the maxillary incisors resulting in most desirable esthetics. Outline form could be determined by drawing the patient face on paper. An esthetic triangle is theory was proposed by Nlelson ${ }^{[1]}$ in which a close relationship between face, tooth, arch was proposed. This theory has bee supported by no of, other works ${ }^{[2,6,7]}$ dentogenics ${ }^{[8,9,10]}$ is a theory derived from observation base on sex personality and age of the patient to produce highly personalized prosthesis and it relies on the sole discretion of the dentist .

Various types of measurement devices have been developed for determining face and tooth size/shape to make selection easy and quick. To determine precisely the degree of correlation between factor a computer technology and formulae have been use but there is no universally accepted single esthetic factor that can be used reliably to aid artificial tooth selection .

The purpose of this article is describe an easy method that incorporate the (basic of the) computer program to analyze the correlation between face form arch form and tooth shape. In this method superimposition of the outline is made to know correlation.

\section{Material And Methods}

50 dental students are selected age group between 20 to 22 yrs male. An impression of each student's maxillary impression was made with irreversible hydrocolloid impression material (Tropical gin, zermack clinic Italy) and study casts were prepared with dental stone . A standardized photographic procedure was adopted to be cord subject's face. In which the camera was mounted on a tripod, was adjusted until it was perpendicular to the subject for and at a distance of $3 \mathrm{ft}$. and two flash unit umbrella were kept to regulate the direct lighting for exposure is carried out for facing photo of cast as well as the central incisor tooth of individual subject selected.

\section{Method}


Outline form and face, Central incisor and arch forms done then for assessing correlation between the these factors. Correlate the esthetic factors by super imposition of the outline of face, central incisor and maxillary arch form is done .all work is done using the paint Photoshop program software.To achieve accurate super imposition the outline, resizing of the images was done with program Photoshop pro 5, which has ability to resize the photo, at the same time maintains the ratio aspect .consequently ,the dental arch was adjusted until the anterior section corresponded in width to the outline of the chin up to the angles of the mandible.

The right central incisor was resized until mesiodistal width of the tooth corresponded with facial width across the zygomas. A precise duplicate of this outline was inverted and transferred into the face for assessment of correlation. In the same way an outline tracing of dental arch was duplicated and inverted on to the face and right central incisor . The superimposition technique in convenient and rapid.

\section{Correlation Of Esthetic Factors.}

Tooth to Face Form :-Gingival margin play a significant role in influencing the dental esthetics, so gingival is used to delineate the tooth neck. In Photoshop program tracing is made around the labial face of the central incisor, which corresponded to mesial and distal contours the incisal edge and cervical margin.

A duplicate of tracing was inverted and transferred on the same subject face to coincide with the chin (cervical margin of gingiva/ tooth) and zygomas (mesial and distal contours) .The incisal edge crossed the forehead and no alteration of the tracing is made for harmony between forms.

Arch to face form

The tracing was made on the arch of the maxillary cast following the incisal edge of anterior and buccal cups of posterior teeth up to the second molors.

A duplicate was then inverted on to the face and curvature is aligned with chin and evaluate for the superimposition status.

Arch to tooth form

A duplicate Tracing of the arch is superimposed on to the central incisor. Here also anterior arch curvature was aligned with the neck of the tooth to assess correspondence of from.A visual Analysis was made to determine the extent \& correlation and findings recorded under on of the three categories.

In all three categories.

1) Correspond: superimposition was done and conformity outline was low as $75 \%$ was sufficient to give very close visual match between factors.

2) Similar: outline tracing that correspond with another factor between $50 \%$ to $75 \%$ of its entirety.

3) Dissimilar, less than $50 \%$ is coincided when superimposition done and visual resemblance between tracing and factor s differed significantly and was negligible.

The totals each group were expressed as percentage to form the result.

\section{Correlation by superimposition}

\section{Results}

The results of superimposition outline form between face tooth , and arch are presented in table I along with the percentage .tooth to face form shows nearly half the cases were dissimilar and only - $\%$ corresponded .This is the lowest level of correspondence in this group . the right corresponded occur between arch to face $\%$.Arch to tooth showed the lower level of dissimilarity at $32 \%$.

Table I : correlation of tooth form to face form $[\mathrm{T} / \mathrm{F}]$. arch form to face form[A/F] and arch form to tooth form $[\mathrm{A} / \mathrm{T}]$ percentage

\begin{tabular}{|l|l|l|}
\hline $\mathrm{T} / \mathrm{F} \mathrm{n}=50$ & $\mathrm{~A} / \mathrm{F} \mathrm{no}=50$ & $\mathrm{~A} / \mathrm{T} \mathrm{n}=50$ \\
\hline
\end{tabular}

\begin{tabular}{|l|l|l|l|l|l|l|}
\hline Category & $\mathrm{n}$ & $\%$ & $\mathrm{n}$ & $\%$ & $\mathrm{n}$ & $\%$ \\
\hline Correspond & 12 & 24 & 16 & 33 & 13 & 26 \\
\hline Similar & 18 & 36 & 17 & 34 & 23 & 46 \\
\hline Dissimilar & 24 & 48 & 18 & 36 & 17 & 34 \\
\hline
\end{tabular}

\section{Discussion}

Although a considerable amount of time was spent in photo graphing of each factor and comprehensive view of the interrelationship between these factors done using Photoshop (software).

A very thin line would have necessitated a more stringent assessment of correspondence. smoothes the contours greater the accuracy was analyzed .The interpretation of outline form was carried out by one person . P.N sellen and etal ${ }^{[11]}$ states correspondence outline through superimposition reveled and insignificant correlation between face, tooth and arch forms. 
Table I shows that approximately one fifth of cases exhibited contumely between face and tooth outline dissimilarity between forms constitutes the largest percentage which is seen in previous studies .Arch to face have show small increase in percentage one tooth to face form but still it represent smallest category.Arch to tooth form shows $24 \%$ of correspondence which reflexes the previous finding in literature The deciding factor is the person's own perception of acceptable similarity between forms. In sufficient to substantiate their use as an reliable esthetical.

\section{Conclusions}

There is insignificant correlation between face, tooth and arch forms. Using this method as an esthetic guide is not advisable and impractical for common application.

\section{References}

[1]. French FA - selection and arrangement of anterior teeth in prosthetic dentures. J. prosthet . dent 1951;1:587-93.

[2]. Schlosser .RO. Complete denture prosthesis $3^{\text {rd }}$ ed. Wb saunders 1953 p-82-97.

[3]. Kvajieck d guides for natural appearance as related to complete denture construction J PROSTHET DENT 1962;21:654-62.

[4]. Boucher co swensons complete dentures $6^{\mathrm{TH}}$ edi cv mo sby : $1970 \mathrm{p}$ 155-94.

[5]. Brodbelt R.H walker GF Nelson D. Comparsion of face shape with tooth form.J prosthet dent 1984;52:588-92.

[6]. Pound E Applying harmony in selecting and arranging teeth dent .clin North AM 1962; march 241-58.

[7]. Hughes GA.facial types and tooth arrangement.J prosthel dent 1951;1:82-95.

[8]. Frush JP, Fisher RD. How dentogenic restorations interpret sex factor, J, proshet dent 1956:6:160-72

[9]. Frush JP fisher RD How dentogenis interprets the personality factor. J prosthet dent 1956:6:441-9

[10]. Frush JD fisher RD factor in Dentogenics J prosthet dent 1957:7:7-13

[11]. P N Sellen ,B Phil.etal.computer generated study of the correlation between tooth face , arch form and palatel contour. J prosthel dent 1998:80:163-168.

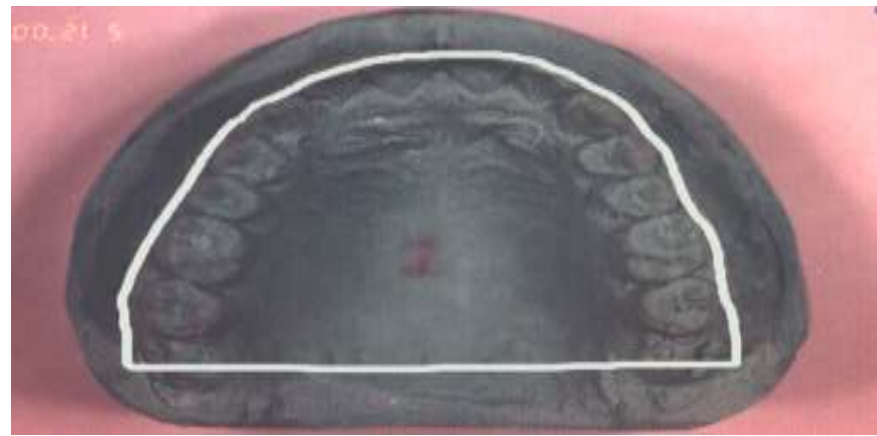

Fig. 1

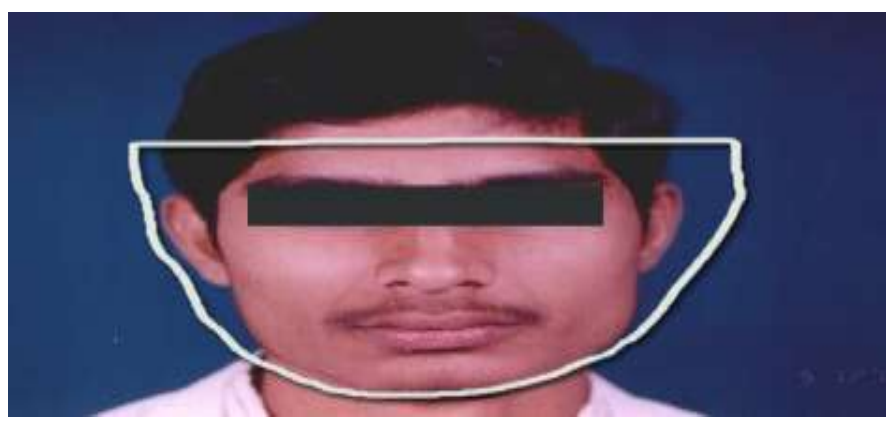

Fig. 2

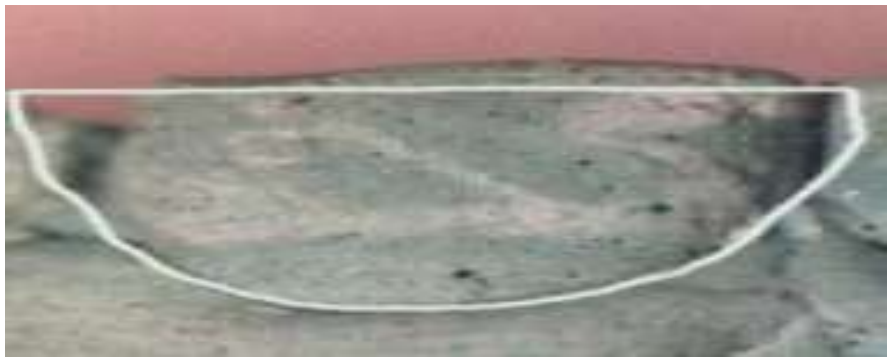

Fig. 3 


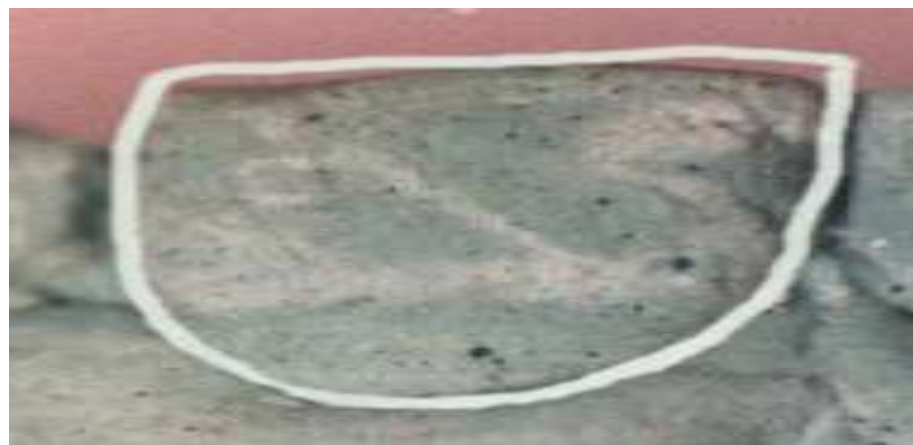

Fig. 4

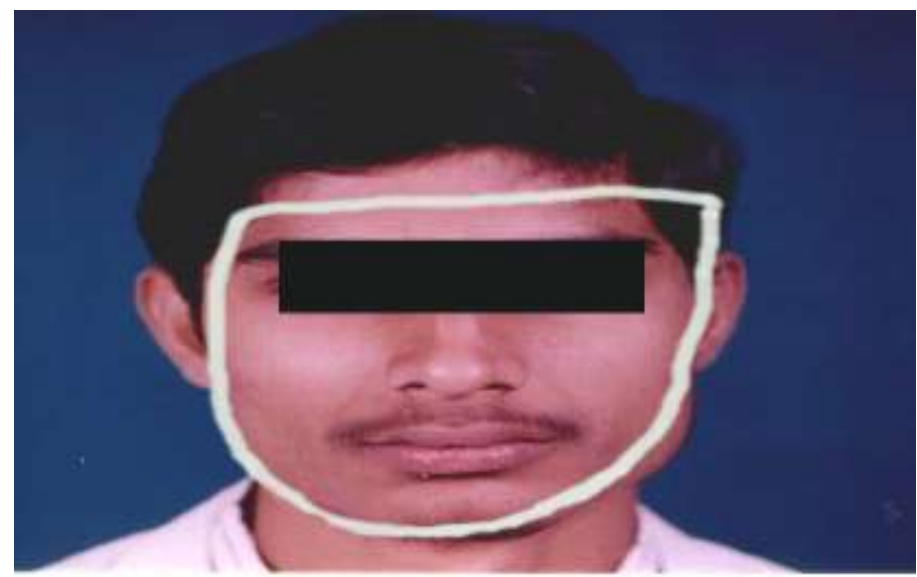

Fig. 5 